\title{
Consommation et modes de vie : une autre perspective sur les politiques d'atténuation du changement climatique
}

\author{
Ghislain Dubois ${ }^{1}$, Jean-Paul Ceron ${ }^{2}$ \\ ${ }^{1}$ Économiste et politologue, TEC, 13001 Marseille, France \\ 2 Économiste, CIRED, 94736 Nogent-sur-Marne cedex, France
}

\author{
Mots-clés : \\ politiques \\ climatiques ; \\ consommation ; \\ mode de vie ; \\ inventaires \\ d'émissions : \\ gaz à effet de serre
}

\begin{abstract}
Résumé - Développées à partir d'une vision macroéconomique et traitant d'abord de la production, les politiques climatiques ont longtemps négligé la question des modes de vie, qui sont pourtant à la fois leur résultante et l'un de leurs leviers d'action majeurs. Leur réintroduction dans les inventaires d'émissions - à la fois par une meilleure analyse des échanges entre branches économiques, et par une déduction des exportations et une inclusion des importations - permet de renouveler le cadre d'analyse. Cette approche par la consommation permet d'envisager des objectifs de négociations internationales basées sur une convergence des émissions par tête en matière de mode de vie, articulés avec des objectifs sectoriels, mais aussi de dessiner de futurs outils comme une TVA carbone. Elle se heurte cependant à des contraintes pratiques de mise en œuvre. À plus court terme, des instruments spécifiques à la sensibilisation et à l'implication des ménages existent, notamment au niveau local. Le présent article vise à fournir les principaux éléments de ces débats sur la base de la littérature récente.
\end{abstract}

\section{Keywords:}

climate policies; consumption; lifestyles; greenhouse gas emissions; inventories

\begin{abstract}
Consumption and lifestyles: an alternative perspective on climate change mitigation policies. Current climate policies were developed from a macro-economic approach in a production perspective, and thus have long neglected the issue of lifestyles. Lifestyles are however the result, and a major factor of greenhouse gas emissions reductions. Their integration in emission inventories, through the deduction of exports and inclusion of imports, along with a better understanding of exchanges between economic branches, allows renewing the analysis framework. This approach helps to envisage climate policies based on a convergence of emissions per capita, expressed in terms of lifestyles, articulated with sectoral production objectives. They also pave the way for tools such as a future "carbon VAT". This is however limited by practical constraints and political acceptability. In the short term, dedicated instruments can help raise awareness and involve households, in particular at the local scale. This paper recalls the main elements of these discussions based on recent literature.
\end{abstract}

\section{Introduction}

\section{Rétablir le citoyen dans les politiques climatiques}

Le citoyen est toujours présent dans les politiques climatiques: en tant que «juge» de la crédibilité des experts - par le biais des sondages -, comme soutien ou frein à l'adoption de nouvelles mesures, mais aussi comme bénéficiaire ou victime du changement climatique et des politiques qui visent à le limiter.
Mais ce citoyen n'est-il pas paradoxalement le grand oublié des politiques climatiques? Les constats scientifiques manient des savoirs complexes dont l'individu peut se sentir exclu, alors qu'il pourrait exister des modalités d'association. L'électeur n'est amené à se prononcer dans les élections que sur des choix à court et moyen terme, quand le changement climatique demande de se projeter à plusieurs dizaines d'années. Les accords sont négociés à une échelle internationale dont l'éloignement du citoyen est notoire.

Auteur correspondant : dubois.ghislain@tec-conseil.com 
Les inventaires d'émissions comme les politiques se sont construites sur le binôme production/territoire, alors qu'il semble qu'on oublie là plusieurs problématiques importantes et les politiques qu'elles inspirent:

- les «fuites de carbone » liées à la délocalisation de la production et au développement des importations, qui donnent une image «trop belle pour être vraie » des trajectoires d'émissions des pays européens ;

- les effets redistributifs des politiques climatiques (la «justice climatique») qui pourraient conduire à des situations de blocage ;

- des leviers d'actions comportementaux qui sont aux mains des individus.

\section{Du micro au macro, et inversement}

Face à ce constat, un certain nombre d'auteurs (voir ci-après) recommandent des approches plus équilibrées entre consommation et production, entre micro et macro, dans la conception des futures politiques. Il s'agit d'assurer un va-et-vient entre les pôles de ces deux échelles, et par là même de considérer à hauteur de son importance la question des modes de vie : partir de «modes de vie durables", construits sur un corps de «bonnes pratiques ", pour les agréger dans des visions collectives, ou désagréger des scénarios macroéconomiques à long terme pour analyser leurs conséquences sur les modes de vie à plus court terme (Gallopin et al., 1997 ; Raskin et al., 2005 ; Anderson et al., 2006 ; Bows et al., 2006 ; Kates et al., 2006; Agence fédérale de l'environnement allemande [Umweltbundesamt], 2009; Rosen et al., 2010 ; Bonduelle et al., 2011 ; EIFER/Sociovision, 2011 ; Dobré, 2012 ; SPREAD, 2012). Et, in fine, définir des régimes climatiques qui combinent des objectifs territoriaux ou sectoriels en matière de production, avec la vision d'une distribution acceptable des émissions par tête liées à la consommation des ménages.

Cet article vise à présenter un ensemble de travaux et de méthodes qui permettent de mieux comprendre la place de l'individu, de sa consommation et plus généralement de ses modes de vie dans le débat climatique, en particulier à partir d'une discussion des inventaires d'émissions. Il essaie ensuite d'imaginer les conséquences pratiques pour les politiques climatiques d'une approche fondée sur la consommation, tant du point de vue des négociations internationales que de l'accompagnement des ménages.

\section{Le besoin d'une perspective de consommation}

Le développement de nouvelles méthodes d'inventaire d'émissions de gaz à effet de serre (GES) qui permettent de répondre au problème des "fuites de carbone» (carbone importé) [Babiker, 2005; Aichele et
Felbermayr, 2012] en réattribuant à un territoire donné l'ensemble des émissions liées à sa consommation (Weber et Matthews, 2008 ; Davis et Caldeira, 2010; Peters et al., 2011) a constitué le point de départ d'une réflexion plus large sur le lien entre consommation et émissions. Ces travaux ont fourni les éléments permettant de comparer des modes de vie et leur empreinte carbone sur des bases solides.

\section{Le renouveau des cadres d'évaluation des émissions}

\section{Un autre regard sur les inventaires}

Les stratégies de réduction de gaz à effet de serre dépendent fortement des évaluations qui sont faites des émissions : il est très clair que jusqu'ici les inventaires des émissions ont été avant tout centrés sur des optiques territoriales et sur la production. Ainsi le cadre de la Convention des Nations unies sur le changement climatique (CNUCC/UNFCCC) inclut les émissions de gaz à effet de serre (et le stockage de ces derniers) dans les territoires nationaux et leurs dépendances (IPCC, 1996). Il comptabilise toutes les émissions de la production à l'intérieur des frontières ainsi qu'une petite partie des émissions de la consommation, c'est-à-dire celles découlant des usages de l'énergie dans l'habitat et de l'usage privé des véhicules à moteur.

La délocalisation des industries polluantes ou tout simplement leur développement dans les pays émergents sont venus relativiser la performance des trajectoires d'émissions (Finke et Gautier, 2013 ; Helm et al., 2007). Le débat sur les «fuites de carbone » prend sa source dans une problématique industrielle de production et de délocalisation, mais en tentant de réintégrer les émissions importées comme l'ont fait plusieurs initiatives présentées dans ce papier, ce sont bien les modes de vie, et donc la consommation qu'on interroge.

\section{Une réflexion sur les périmètres des inventaires}

Différentes optiques peuvent présider à la construction des inventaires d'émissions, éclairant de manière différente les stratégies d'atténuation. L'intérêt de ces démarches peut d'abord être mis en évidence par une typologie des émissions: dans le tableau ci-aprés figurent en colonne la localisation de la production ou de la consommation, et en ligne la propriété des installations de production et la nationalité des consommateurs.

On notera que les catégories 4,8 et 12 ne sont pas pertinentes pour des inventaires nationaux. Les inventaires nationaux tels que définis par la CNUCC comprennent les catégories 1 et 3 ainsi qu'une partie des 
Tableau. Les différents éléments des inventaires nationaux d'émissions.

\begin{tabular}{|c|c|c|c|c|}
\hline & & & \multicolumn{2}{|c|}{ Location of production or consumption } \\
\hline & & & Domestic & Abroad \\
\hline \multirow[t]{2}{*}{$\begin{array}{l}\text { Ownership } \\
\text { of production } \\
\text { facilities }\end{array}$} & \multicolumn{2}{|c|}{ National } & $\begin{array}{l}\text { 1. Inland emissions from national } \\
\text { production facilities }\end{array}$ & $\begin{array}{l}\text { 2. Emissions abroad from national } \\
\text { production facilities and international } \\
\text { shipping }\end{array}$ \\
\hline & \multicolumn{2}{|c|}{ Foreign } & $\begin{array}{l}\text { 3. Inland emissions from production } \\
\text { facilities owned by foreigners }\end{array}$ & $\begin{array}{l}\text { 4. Emissions abroad from production } \\
\text { facilities owned by foreigners }\end{array}$ \\
\hline \multirow[t]{4}{*}{$\begin{array}{l}\text { Nationality } \\
\text { of consumer }\end{array}$} & \multirow{2}{*}{$\begin{array}{l}\text { Products and } \\
\text { services } \\
\text { (p\&s) } \\
\text { produced } \\
\text { inland }\end{array}$} & National & $\begin{array}{l}\text { 5. Inland emissions from national } \\
\text { consumption of p\&s produced inland }\end{array}$ & $\begin{array}{l}\text { 6. Emissions abroad from national } \\
\text { consumption of p\&s produced inland }\end{array}$ \\
\hline & & Foreign & $\begin{array}{l}\text { 7. Inland emissions from consumption } \\
\text { by foreigners of p\&s produced inland }\end{array}$ & $\begin{array}{l}\text { 8. Emissions abroad from consumption } \\
\text { by foreigners of p\&s produced inland }\end{array}$ \\
\hline & \multirow{2}{*}{$\begin{array}{l}\text { Products and } \\
\text { services } \\
\text { (p\&s) } \\
\text { produced } \\
\text { abroad }\end{array}$} & National & $\begin{array}{l}\text { 9. Inland emissions from national } \\
\text { consumption of p\&s produced abroad }\end{array}$ & $\begin{array}{l}\text { 10. Emissions abroad from national } \\
\text { consumption of p\&s produced abroad }\end{array}$ \\
\hline & & Foreign & $\begin{array}{l}\text { 11. Inland emissions from consumption } \\
\text { by foreigners of p\&s produced abroad }\end{array}$ & $\begin{array}{l}\text { 12. Emissions abroad from consumption } \\
\text { by foreigners of p\&s produced abroad }\end{array}$ \\
\hline
\end{tabular}

Source : Aall et Hille (2009).

sources d'émissions contenue dans les catégories 5 et 7 (logement et usage privé de véhicules motorisés). Un inventaire des émissions concernant strictement la production inclurait les catégories 1,2 et 3 . Symétriquement, un inventaire des émissions de la consommation inclurait les catégories 5 à 11 (à l'exception de la catégorie 8). Ceci peut souffrir des exceptions sectorielles, par exemple quand on considère le tourisme, pour lequel on pourrait inclure les émissions du transport des touristes étrangers à destination et à partir du pays concerné (catégories 8 et 12), à côté des émissions des résidents pendant leurs vacances à l'étranger (catégories 6 et 10) [Aall et Hille, 2009].

\section{Les insuffisances d'une optique centrée sur la production}

On peut d'abord rappeler que la production et donc ses émissions sont sous-tendues par des pratiques de consommation et des modes de vie qui expliquent leur dynamique (voir en particulier la synthèse bibliographique effectuée dans les chapitres 4 et 5 du groupe de travail III du GIEC [IPCC, 2014, pp. 307-308 et 387-389]). Tant au niveau mondial que pour la France ou le Royaume-Uni, on a ainsi pu montrer que les choix des consommateurs déterminent ou influencent les trois quarts des émissions nationales (Wiedmann et al., 2008 ; Lenglart et al., 2010, p. 101 ; Edgar et Glen, 2009, p. 6414).

Le Groupement d'experts intergouvernemental sur l'évolution du climat (GIEC) reconnaît d'ailleurs depuis longtemps le besoin de prendre en compte la lutte contre les émissions potentielles des systèmes d'activités socioéconomiques, parallèlement à celles de secteurs économiques comme l'énergie, l'agriculture et le transport (IPCC, 2014). La consommation a été peu évoquée dans le quatrième rapport du GIEC ; le cinquième a proposé une double approche des inventaires (production et consommation, après déduction des exportations et réintroduction des importations) [IPCC 2014, pp. 305-306], sans prendre en compte la dimension des modes de vie à hauteur de leur importance. Le rapport du GIEC (chapitres 4 et 5) adopte avant tout une entrée par les comportements (behaviour) : pourquoi les individus n'adoptent-ils pas les solutions mises à disposition par la technique et l'économie? L'économie des comportements (behavioural economics) [chapitre 5, p. 388] est censée contribuer à lever ces obstacles (barriers) ; on est largement dans le registre d'une vision utilitariste, voire manipulatrice, de la science comme le fait remarquer la sociologue E. Shove (2010). En tout état de cause, on est loin de prendre la question des modes de vie à bras-lecorps, ce qui aurait pu se faire par exemple en consacrant un chapitre à la consommation et aux modes de vie à hauteur de ce qui se fait pour les chapitres sectoriels (agriculture, etc.). Une illustration de cet état de fait peut être fournie par l'expérience d'un des deux auteurs de cet article dans le cinquième rapport du GIEC (groupe III). Le thème qu'il était chargé de traiter, le tourisme, a été attribué au chapitre 10 (industrie). On peut certes rencontrer le terme "d'industrie touristique", mais réduire une pratique sociale complexe à une partie de sa dimension marchande témoigne bien de la difficulté à aborder les modes de vie. L'article de Shove (2010) montre que cela est lié à la faible présence de disciplines telles que la sociologie, l'anthropologie, la psy- 


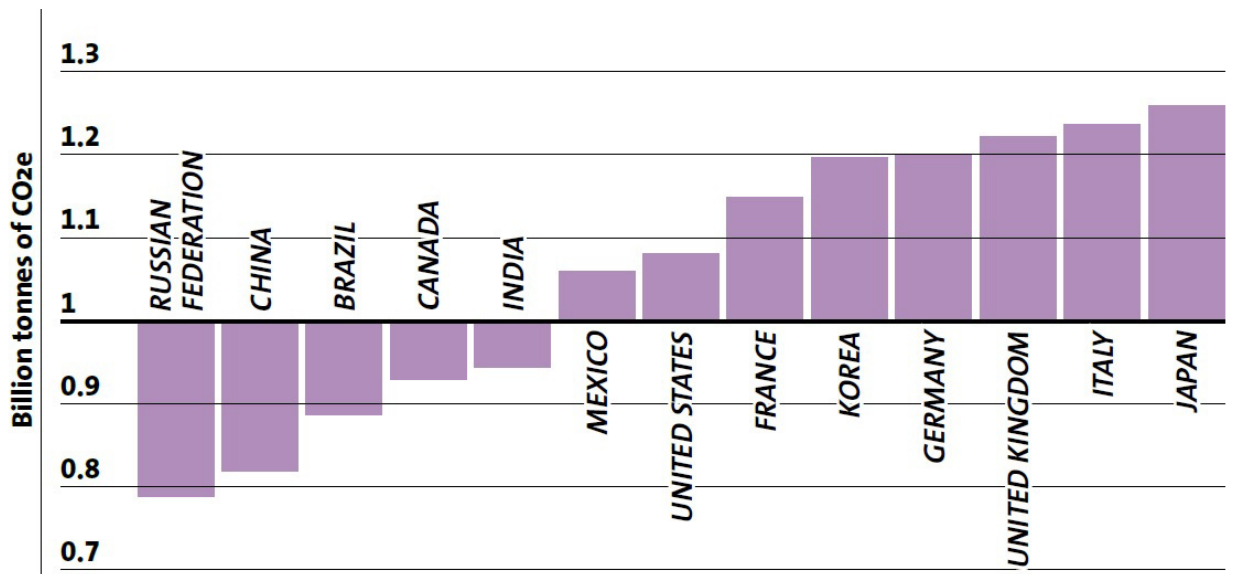

Fig. 1. Écart entre les inventaires fondés sur la production et ceux sur la consommation pour les 13 principaux pays émetteurs (Munasinghe et al., 2009, p. 18). Analyse du Stockholm Environment Institute, données fournies par Hertwich et Peters (2009).

chologie, etc. face aux sciences exactes et à l'économie dans la construction du savoir sur le changement climatique. Toutefois il faut aussi noter qu'entre le quatrième et cinquième rapport du GIEC l'approche d'un chapitre aussi ancré sectoriellement que l'industrie a évolué. On ne s'y préoccupe plus seulement des processus de fabrication et de leurs émissions, mais aussi des modes d'usage des produits et de l'organisation des services qu'ils rendent (IPCC, 2014, pp. 745-746), ce qui constitue un pas en avant très significatif.

L'approche par la production des émissions de gaz à effet de serre revient à faire porter à chaque pays la responsabilité des GES inclus dans ses exportations, et le dédouane de toute responsabilité liée au $\mathrm{CO}_{2}$ inclus dans ses importations. Pour la France, en 2005, les émissions $\mathrm{du}$ seul $\mathrm{CO}_{2}$, induites par l'activité économique sur le territoire français sont de 410 millions de tonnes, soit 6,7 tonnes par an et par habitant. Les émissions de $\mathrm{CO}_{2}$ de la demande finale intérieure française sont de 545 millions de tonnes, soit 9 tonnes par an et par habitant, dont 40 \% dus à nos importations (Lenglart et al., 2010).

Si l'on introduisait ce correctif pour les États-Unis par exemple, on s'apercevrait que les responsabilités en matière d'émissions de gaz à effet de serre de ce pays sont supérieures de $8 \%$ par rapport à la comptabilité de type CNUCC (Munasinghe et al., 2009, p. 5). À l'inverse environ $20 \%$ de la croissance des émissions des pays «non-annexe $\mathrm{B}^{1}$ » résultent de leur production exportée (Peters et al., 2011).

Le poids des gaz à effet de serre dans les exportations ne concerne pas uniquement les pays émergents. Comment évaluer la responsabilité des pays producteurs de pétrole ou plus généralement exportateurs d'hydrocarbures ou de charbon dans la pollution qu'ils génèrent (cela concerne non seulement des pays

\footnotetext{
1 En développement et émergents.
}

producteurs du Sud, mais également des pays du Nord comme la Norvège) ? Enfin, le $\mathrm{CO}_{2}$ n'est pas le seul gaz à considérer. Les pays du Nord importent ainsi du $\mathrm{CH}_{4}$ avec leurs importations de produits animaux ou de riz, à tel point que ce $\mathrm{CH}_{4}$ importé est dans la plupart des cas supérieur à leurs émissions nationales de ce gaz (Peters et Hertwich, 2006, p. 379 ; Subak, 1995). L'écart entre les deux modes de comptabilité (en équivalent $\mathrm{CO}_{2}$ ) est illustré dans la figure 1 pour les treize plus gros pays émetteurs.

Ce type de constatations n'est pas récent et date des années 1990. Il est également clair que le solde de la balance import/export de GES a beaucoup évolué au cours des trente dernières années avec une accélération récente de la dégradation dans les pays du Nord. Ainsi le Danemark connaissait en 1987 un surplus de 0,5 million de tonnes qui s'est mué en un déficit de 7 millions de tonnes en 1994 (Frese et al., 2008). La désindustrialisation des pays du Nord est manifestement en cause.

\section{De la consommation aux modes de vie}

\section{Le périmètre d'une optique de consommation}

Une vision intégrale des émissions liées à la consommation demande :

- de prendre en compte la consommation des résidents à l'étranger (par ex. pendant des voyages d'affaires ou des vacances) et de déduire celle des non-résidents en France ;

- d'appréhender les émissions liées à la consommation qui se situent hors de toutes frontières, par exemple par l'intermédiaire de l'aviation et du transport maritime ; - de prendre en compte la consommation de gaz à effet de serre incluse dans les produits importés et de déduire les exportations (Helm et al., 2007, p. 17) ; 


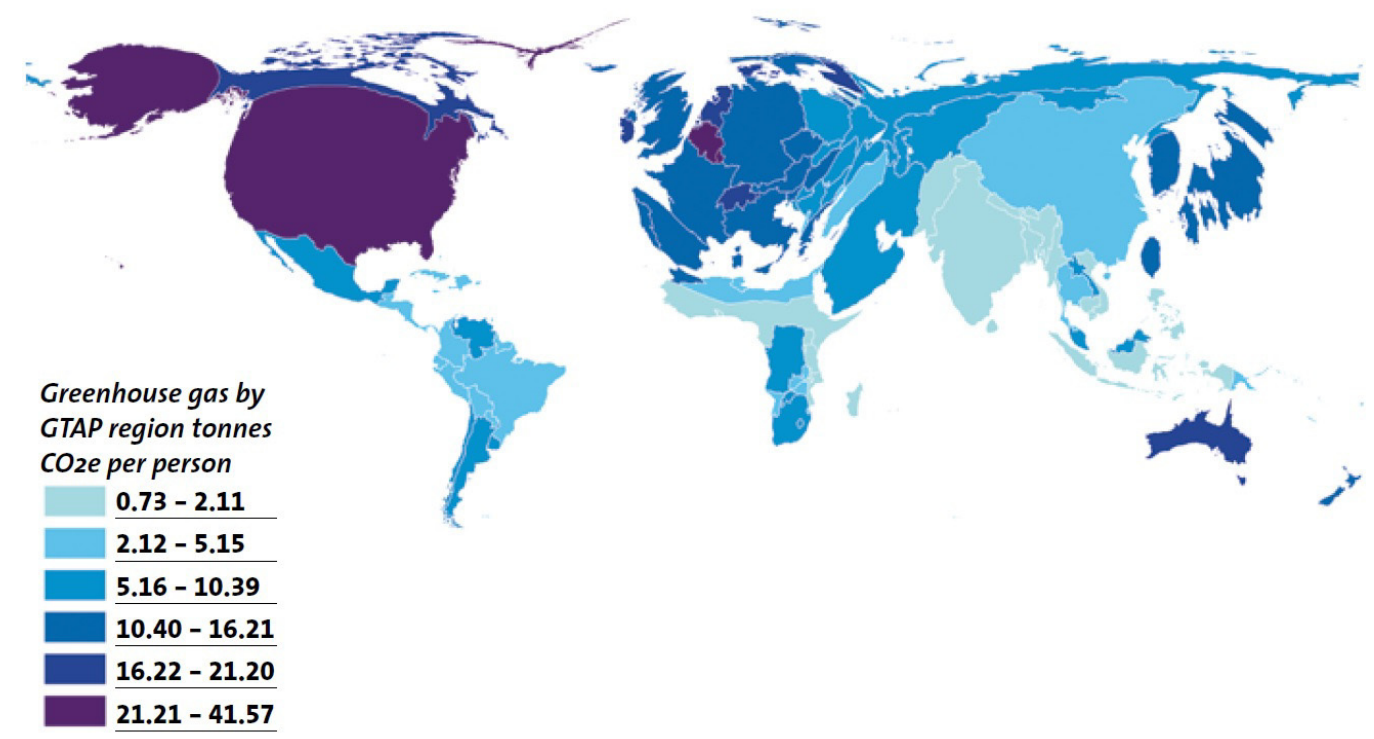

Fig. 2. Carte du monde déformée selon les émissions de la consommation des pays (Munasinghe et al., 2009, p.17).

- de mieux appréhender la distribution sociale des émissions, en raison de la variabilité des situations et des comportements individuels à l'intérieur d'un groupe social.

La mise en œuvre de ces objectifs se heurte à des difficultés soulignées dans le chapitre 5 du rapport du GIEC (IPCC, 2014, p. 364) : aux incertitudes des émissions calculées sur une base territoriale (toujours utilisées dans une optique de consommation) s'ajoutent celles des statistiques sur le commerce international et surtout les incertitudes dans les définitions, les niveaux d'agrégation et les hypothèses de calcul (Kanemoto et al., 2012).

\section{Une vision globale}

Le travail de Munasinghe et al. (2009) a tenté de donner une perspective globale, en calculant les émissions par tête dans une optique de consommation. Parallèlement à la taille déformée des pays, les couleurs de la figure 2 rendent compte de la consommation et des émissions par tête. De ce point de vue, il est clair que la seule raison de l'importance de la Chine et de l'Inde est leur population considérable. Les États-Unis, le Canada, l'Australie, les Pays-Bas et la Belgique ont les émissions par habitant les plus élevées et sont suivis par la plupart des autres pays de l’Union européenne.

Une vision des inégalités dans la répartition des émissions a été donnée par Chakravartya et al. (2009). Elle introduit l'idée, pas forcément facile à mettre en pratique, de politiques climatiques qui visent les ménages les plus émetteurs, quel que soit leur pays d'appartenance. La figure 3 montre la part cumulée des habitants et la part décroissante cumulée des émissions. L'aire totale sous la courbe correspond aux émissions totales en 2030 dans un scénario business as usual $\left(43 \mathrm{GtCO}_{2}\right)$ et l'aire grisée montre les $13 \mathrm{GtCO}_{2}$ qui devraient être retirés pour atteindre les $30 \mathrm{GtCO}_{2}$ (noté « 30 ») visés. Le taux d'émission individuelle nécessaire pour retirer ces $13 \mathrm{GtCO}_{2}$ sans affecter les autres est de $10,8 \mathrm{t}$ de $\mathrm{CO}_{2}$ par tête. Quelque 1,13 milliard de personnes dépassent cette limite dans le monde. Sont aussi illustrés le taux d'émission par tête nécessaire pour atteindre une cible d'émission de $20 \mathrm{GtCO}_{2}$ (4,9 tonnes de $\mathrm{CO}_{2}$ par tête), $25 \mathrm{GtCO}_{2}$ (7,3 tonnes par tête). La courbe de 2030 est par ailleurs comparée avec celle de 2003.

Le carbone inclus dans le commerce extérieur a fait l'objet d'études approfondies dans un certain nombre de pays : le Royaume-Uni (Helm et al., 2007 ; Wiedmann et al., 2010), la Suède (Eriksson, 2008), la Finlande (Mäenpää et Siikavirta, 2007), la Chine (Hong et al., 2007), 1'Autriche (Muñoz et Steininger, 2010). Nous développons plus longuement ci-dessous le cas de la France.

\section{Le cas de la France}

Le service de l'observation et des statistiques (SOeS) du ministère de l'Écologie a engagé un programme de travail visant à faire le lien entre l'émission d'un gaz à effet de serre et chaque activité productive, du point de vue du consommateur final et de celui du producteur. L'Insee fournit les données de comptabilité nationale et le Citepa, les comptes physiques d'émissions atmosphériques par activités économiques. Une première publication (Pasquier, 2010) a décrit les émissions de $\mathrm{CO}_{2}$ en France selon les acteurs (ménages, entreprises et 


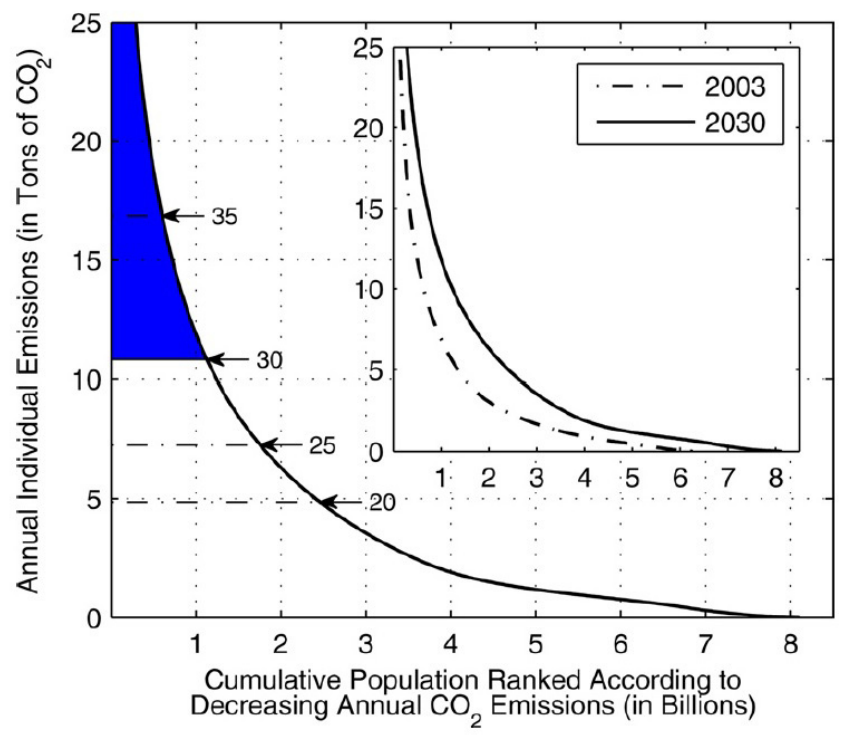

Fig. 3. Répartition de la population mondiale selon ses émissions annuelles par tête. L'axe des ordonnées représentent les émissions annuelles par tête et l'axe des abscisse la population mondiale cumulée en fonction de ses émissions par tête décroissantes [Chakravartya et al., 2009, p. 17].

administrations publiques) et les activités économiques (production, consommation), et identifie le niveau des émissions de $\mathrm{CO}_{2}$ associées à la demande intérieure française, y compris celles émises à l'étranger via les importations (CGDD, 2010 ; Lenglart et al., 2010).

\section{Consommation et modes de vie}

Près de 410 millions de tonnes de $\mathrm{CO}_{2}$ étaient émises sur le territoire national en 2005. Quelque 130 sont constituées par les émissions directes des ménages, pour part sensiblement égale, issues des carburants des transports et $\mathrm{du}$ fioul et du gaz destinés au chauffage des habitations. Environ 280 millions de tonnes sont émises par la production de biens et de services, dont les deux tiers liés à la demande finale intérieure et un tiers aux exportations.

Tout d'abord, le cadre de la comptabilité nationale permet de décrire les échanges entre branches et, chaque unité de valeur monétaire étant affectée d'un coefficient d'émission de $\mathrm{CO}_{2}$ (source Citepa), de procéder à l'affectation des émissions à la demande finale. Sur les 280 millions de tonnes, la moitié correspond à des consommations intermédiaires ainsi réaffectées. Il en résulte que certaines branches voient leurs émissions réduites alors que d'autres augmentent significativement, comme l'automobile (Fig. 5).

Ensuite, le $\mathrm{CO}_{2}$ caché dans la consommation finale ne trouve pas son origine uniquement sur le territoire national. Une fois réalisée la réaffectation décrite ci-dessus, il est nécessaire d'imputer à la demande finale le $\mathrm{CO}_{2}$ contenu dans les importations. Ceci concerne les biens achetés directement par le consommateur (une télévision ou un ordinateur importés, etc.) incluant le contenu en $\mathrm{CO}_{2}$ des biens intermédiaires qui ont servi à les fabriquer. Cela concerne aussi le contenu en $\mathrm{CO}_{2}$ des biens intermédiaires importés d'un produit fabriqué en France (composants importés d'une automobile par exemple). Il faut également tenir compte du fait qu'une partie du $\mathrm{CO}_{2}$ que la France importe « repart " à l'étranger par le truchement des produits exportés qu'il a servi à fabriquer (110 MT à rajouter aux 95 MT d'émissions liées à l'exportation sur le territoire national) [Lenglart et al., 2010, p. 111].

Une fois les réaffectations effectuées, il faut, dans une optique consommation, retrancher aux émissions produites sur le territoire celles liées aux exportations et rajouter celles des importations. La figure 4 exprime les résultats de cette démarche.

La quantité de $\mathrm{CO}_{2}$ associée à la demande finale est donc de 545 MT dont un peu plus de $40 \%$ sont émis à l'étranger. Sur une base territoriale, un Français paraît responsable de 6,7 t de $\mathrm{CO}_{2}$, dans une optique consommation, c'est $9 \mathrm{t}$. La figure 5 présente la ventilation des émissions par activités et usages.

Le tableau sera encore noirci si on ne se contente pas d'une analyse en termes de $\mathrm{CO}_{2}$. L'analyse a en effet été complétée en ajoutant au $\mathrm{CO}_{2}$, le $\mathrm{CH}_{4}$ et le $\mathrm{N}_{2} \mathrm{O}$ de façon à prendre en compte l'essentiel des GES. Il en résulte que le passage du $\mathrm{CO}_{2}$ au $\mathrm{CO}_{2}$-équivalent se traduit pas une augmentation d'un tiers des émissions par tête, dans une optique de consommation. 


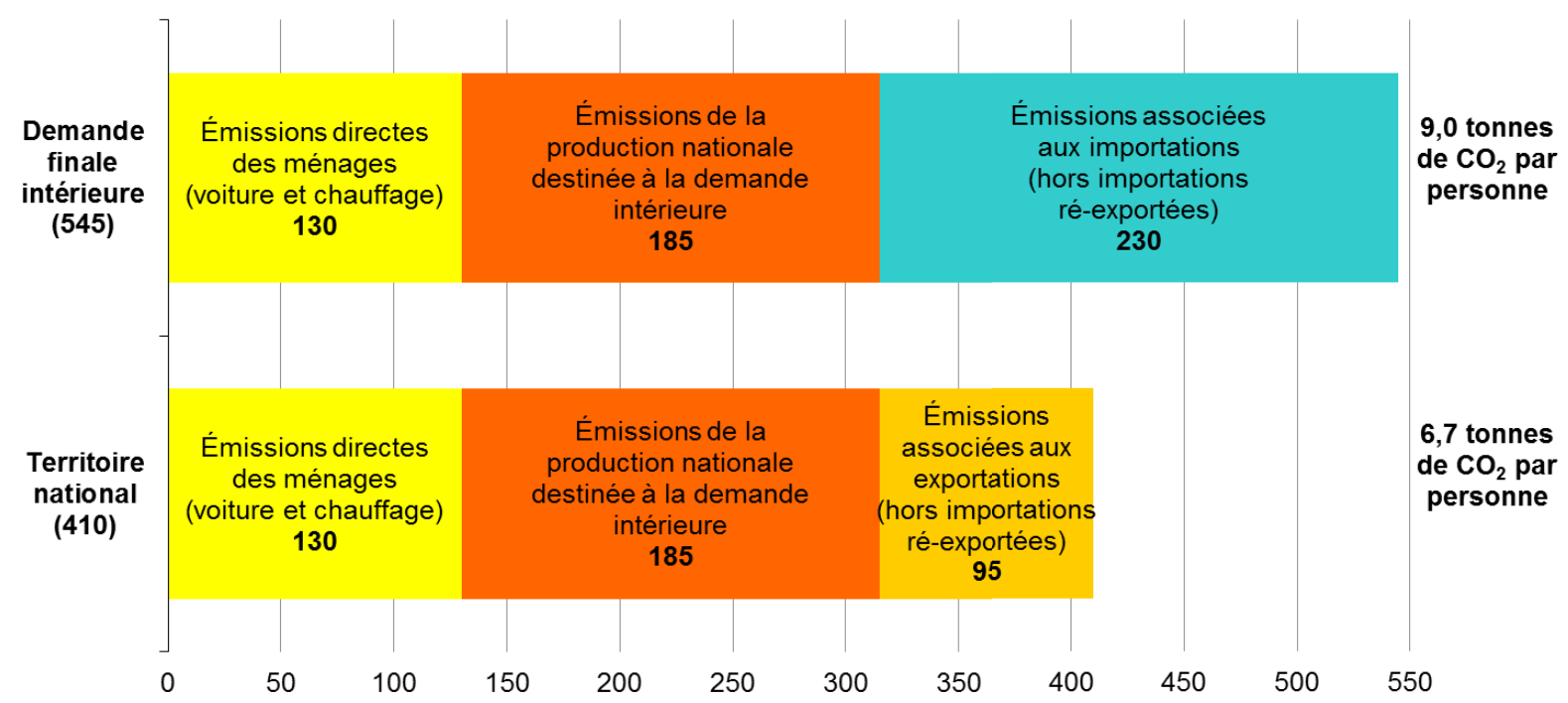

Notes : $\mathrm{CO}_{2}$ émis sur le territoire de la France métropolitaine en 2005 , hors $\mathrm{CO}_{2}$ issu de la combustion de biomasse à des fins énergétiques et hors utilisation des terres, leur changement et la forêt (UTCF) ; Emissions des importations ré-exportées : générées à l'étranger du fait du contenu en importations des exportations françaises.

Sources : SOeS, calculs d'après Citepa - Insee - Douanes - Eurostat et AIE, 2010

Fig. 4. Empreinte carbone de la demande finale intérieure de la France versus émissions sur le territoire national (source : ministère de l'Écologie).

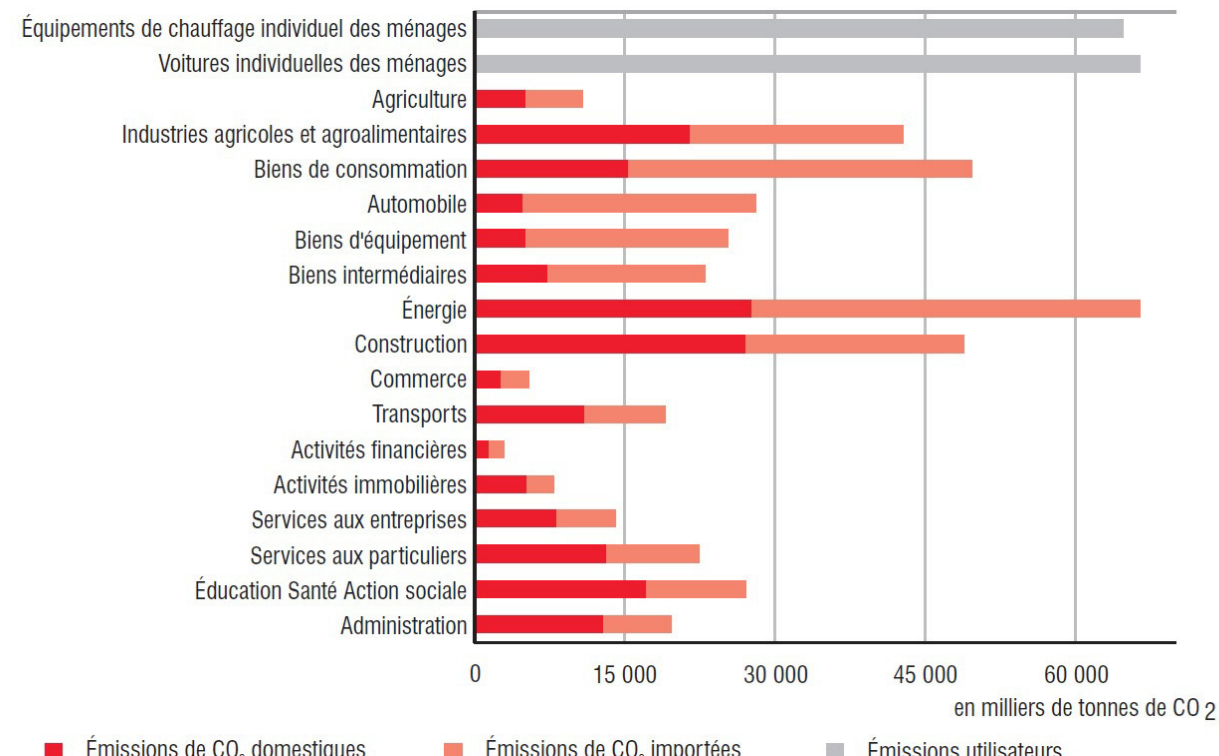

Fig. 5. Émissions de $\mathrm{CO}_{2}$ domestiques et importées induites par la demande finale intérieure (2005) [Lenglart et al., 2010]. Lecture : la demande finale en construction induit 49 millions de tonnes de $\mathrm{CO}_{2}$, dont 27 émises par l'appareil de production domestique et 22 émises à l'étranger via les importations de produits utilisés pour répondre à cette demande finale.

\section{Inégalités et justice carbone}

En zoomant encore, il est possible, après être passé de la production à la demande finale au niveau macro, d'atteindre une définition plus fine de la responsabilité des ménages au niveau micro.
La demande finale recouvre en effet en France trois éléments (Pasquier, 2010) :

- les dépenses de consommation des ménages (75\% des émissions pour $55 \%$ du PIB) ;

- les dépenses de consommation des administrations publiques (10\% des émissions pour $25 \%$ du PIB) ; 

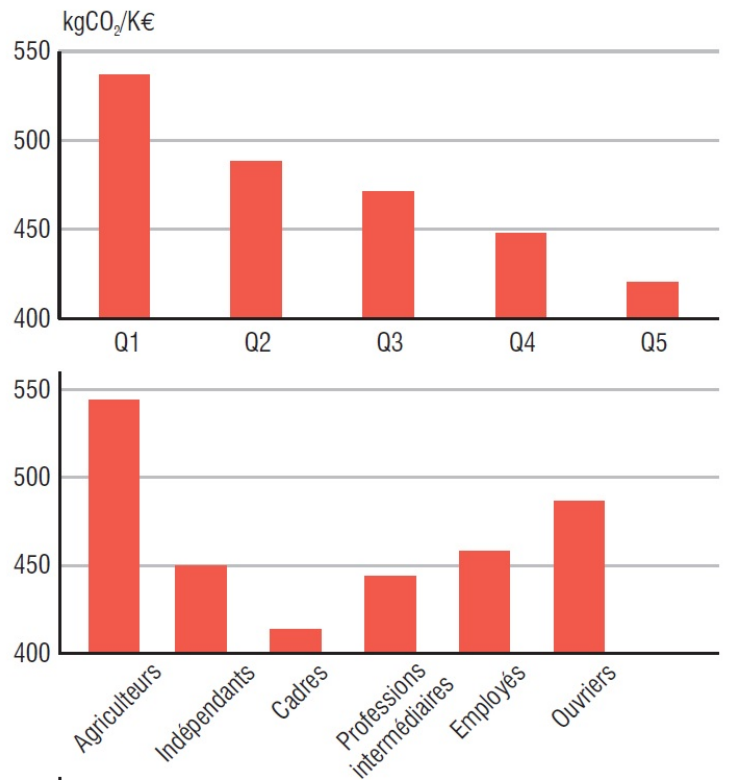

Fig. 6. Propension à émettre du $\mathrm{CO}_{2}$ (ratio émission de $\mathrm{CO}_{2}$ sur dépenses de consommation) selon les catégories de ménages par quintile de niveau de vie (haut) et par catégorie socioprofessionnelle (bas) [Lenglart et al., 2010].

- l'investissement (15\% des émissions pour $20 \%$ du PIB).

Au niveau des ménages, il est possible d'approcher la question des inégalités dans la distribution des émissions. On montre par exemple que les émissions des ménages croissent avec le niveau de vie : le quintile des ménages les plus aisés rend compte de $29 \%$ des émissions de $\mathrm{CO}_{2}$, alors que le quintile des plus modestes n'est responsable que de $11 \%$. L'écart de 1 à 2,7 est inférieur à celui de 1 à 3,4 qui caractérise leurs niveaux de consommation respectifs, ce qui signifie que le contenu en $\mathrm{CO}_{2}$ par euro dépensé (Fig. 6) est, lui, décroissant avec le niveau de vie (Lenglart et al., 2010, p. 113). Cela dénote également une plus grande vulnérabilité des ménages modestes à la hausse des prix de l'énergie ou aux politiques de lutte contre l'effet de serre comme l'avaient suggéré les études menées lors de la dernière hausse des prix du pétrole : le contenu en $\mathrm{CO}_{2}$ par euro dépensé par un ménage agriculteur ou ouvrier est plus important que celui d'un ménage cadre (respectivement de $32 \%$ et de $18 \%$ ). Ceci s'explique par plusieurs facteurs, mais d'abord par le fait qu'en dépit d'une part des dépenses de logement dans le budget de consommation d'environ un quart quel que soit le niveau de vie, la part dédiée aux charges (incluant le chauffage particulièrement émetteur de $\mathrm{CO}_{2}$ ) compte pour $9 \%$ du budget pour le quintile des ménages les plus modestes et $4 \%$ pour les plus aisés (Lenglart et al., 2010, p. 114). Une connaissance fine de la répartition des émissions entre les consommateurs est donc utile pour ajuster les politiques d'atténuation et leurs effets sociaux.

\section{Les conséquences possibles d'une perspective de consommation}

Il est possible de se demander quelles pourraient être les conséquences pratiques sur les politiques climatiques d'une perspective de consommation, ou tout au moins $\mathrm{d}$ 'approches plus équilibrées combinant production et consommation.

Sur le fond, cette interrogation a deux sources. De manière pragmatique, elle vise à prendre acte du fait que puisque les mesures prises dans le cadre des politiques d'atténuation (taxes carbone ou permis négociables pour les industriels par exemple) auront de toute façon des impacts sur les ménages - notamment par l'intermédiaire d'un effet sur les prix - autant s'en préoccuper en amont. D'où le besoin d'une vision globale de l'impact de ces politiques sur les modes de vie (déclinaison de scénarios globaux, analyse de l'impact des accords internationaux), et d'un accompagnement des ménages dans la transition (outils «micro»), pour éviter les blocages ou les iniquités insupportables.

Plus fondamentalement, cette interrogation correspond à un «moment » des politiques climatiques où l'on se rend compte que l'on ne pourra pas éviter de toucher aux modes de vie. Ni la technologie ni l'adaptation au fil de l'eau des entreprises ne permettront d'atteindre 65 à $80 \%$ de réduction des émissions mondiales préconisées par le GIEC pour rester dans la limite des $+2{ }^{\circ} \mathrm{C}$ (Parry et al., 2008 ; Parry et Palutikov, 2008 ; Meinhausen, 2006 ; Hansen et al., 2008) et des adaptations des modes 
de consommation - sinon des renoncements - seront nécessaires. Il peut d'ailleurs sembler surprenant que ce constat mette tant de temps à être accepté au-delà des cercles militants. Difficulté du renoncement? Réflexes technocratiques refusant d'aller au-delà des solutions les plus évidentes et acceptables ? La célèbre phrase de George Bush au sommet de Rio en 1992, «Le mode de vie américain n'est pas négociable », reste un leitmotiv pour beaucoup.

Sur la forme, ces conséquences sur les politiques climatiques peuvent être classées selon deux critères : leur échelle de mise en œuvre (niveau micro des ménages, ou niveau macro des politiques) et leur mode de mise en œuvre, réglementaire ou volontaire.

\section{Les politiques domestiques et internationales}

L'objectif n'est pas ici de proposer une refondation des régimes de négociation, mais, très en amont, de réfléchir sur les implications possibles de la fixation éventuelle d'objectifs de réduction d'émissions de GES exprimés en termes de consommation, sur les outils qui permettraient d'accompagner cette vision, tout en mettant déjà en évidence quelques limites. La réflexion est donc totalement a-juridique, c'est-à-dire déconnectée du protocole de Kyoto ou de tout autre instrument réglementaire. On n'examine pas dans un premier temps les difficultés de mise en place de cette orientation.

\section{Les termes du débat}

Les émissions importées ne seraient pas nécessairement un problème dans le cadre de politiques climatiques globales (Steckel et al., 2010), mais le sont en régime partiel ou fragmenté (Peters et Hertwich, 2008 ; Bows et Barrett, 2010), quand une partie simplement des entreprises et des États sont soumis à des objectifs de réduction. Cette volonté de traiter les «fuites de carbone ", et par là même de reconstituer l'empreinte carbone des modes de vie des habitants d'un pays, a incité à développer une perspective de consommation.

Cependant, réintégrer les importations dans un inventaire d'émissions ne règle pas tout. Il faut ensuite trancher plusieurs problèmes qui conditionnent l'optimisation des émissions.

- Faut-il différencier les catégories de biens ou de matières premières énergétiques pour tenir compte du cas où les émissions exportées sont largement supérieures à celles qui sont émises sur le site de production? C'est le cas par exemple des produits pétroliers, comptabilisés aujourd'hui principalement sur leur lieu de consommation. Dans ce cas, l'existence d'une « rente » doit-elle entraîner aussi une responsabilité du producteur?

- Qui profite de quoi et sur qui doit peser la responsabilité ? Le producteur qui en tire salaires et profits, ou le consommateur qui utilise le bien produit? L'argument, loin d'être théorique, est souvent utilisé par les négociateurs, la Chine insistant à Copenhague sur le fait que finalement elle produit et pollue en partie pour les pays développés. Comment répartir cette responsabilité évidemment partagée ? On pense ici aux émissions de la production, mais aussi à celles du transport international, maritime ou aérien. Faut-il l'attribuer à un pays en particulier, aux deux à $50 \%$, ou ne garder qu'une régulation internationale à travers des accords sectoriels ?

- Où produire ? Quel mode de fixation des objectifs est susceptible de mener à une division internationale du travail optimisant les émissions de gaz à effet de serre associées ? La Nouvelle-Calédonie est par exemple souvent citée comme un territoire dont les émissions par tête dépassent celles des États-Unis. Ceci n'est pas particulièrement dû au mode de vie de ses habitants, mais à la structure de son économie, dominée par la production de nickel, très énergivore (Ceron et al., 2010). Une régulation fondée sur des objectifs territoriaux, basés sur la production, conduirait à décourager la production de nickel, en soumettant la Nouvelle-Calédonie à une contrainte très forte. Pourtant, on sait que le monde a besoin de nickel et qu'il est en principe plus efficace d'un point de vue énergétique de transformer le minerai près de son lieu de production. Du point de vue de l'efficacité globale en matière de lutte contre les émissions de GES, cela tend à suggérer de réguler les émissions de nickel dans le cadre d'un schéma international basé sur la production, par exemple un mécanisme d'échanges de permis entre industriels dans un cadre global ou sectoriel, les autres sources d'émissions, plus dépendantes des ménages, étant régulées sur la base d'inventaires de consommation.

\section{Renouveler le cadre de la fixation des objectifs nationaux}

Une fois ces problèmes posés (mais non résolus), il est possible d'imaginer plusieurs types de régulation:

- des objectifs nationaux qui seraient uniquement basés sur la consommation, la charge intégrale des importations étant attribuée au pays importateur ;

- le régime mixte actuel, fondé en majorité sur la production, avec une prise en compte de la consommation dans le cas des produits pétroliers importés ;

- un autre régime mixte, plus tourné vers la consommation : les objectifs «territoriaux" seraient d'abord fixés dans une optique de consommation, et les émissions des grands secteurs industriels seraient régulées sur la base d'accords sectoriels (accords réglementaires ou volontaires), par exemple dans le cadre de mécanismes d'échanges de permis.

Dans les deux derniers cas, une des difficultés est d'éviter une double taxation des émissions. Taxer 
doublement peut cependant permettre de partager la charge entre producteur et consommateur, ce qui pourrait être empiriquement acceptable si le prix du carbone n'est pas trop élevé, même si ce n'est guère satisfaisant au plan théorique.

Une première conséquence d'une optique de consommation serait de durcir les niveaux de réduction demandés à la plupart des pays développés. Pour la France, par exemple, si l'on veut atteindre un facteur 4 en 2050 (soit $75 \%$ de réduction) - ce qui est toujours l'objectif officiel calculé sur la base de 6,7 t en 2005-, le droit à émettre est de $1675 \mathrm{t}$; reformulé en termes de demande finale (base $9 \mathrm{t}$ ), c'est en fait une réduction de $81,4 \%$ qu'il faudrait réaliser.

\section{Vers une TVA carbone?}

L'idée d'une "TVA carbone », en lieu et place d'une taxe carbone, pourrait être un moyen de déployer une approche de consommation ${ }^{2}$ :

- Elle constitue un moyen de responsabiliser les individus, en impactant directement leur budget.

- Elle permet de traiter l'ensemble des biens et services, et pas seulement les biens produits sur le territoire, et donc d'opérer un possible " ajustement aux frontières ". - Elle pourrait également être un levier incitatif fort, avec par exemple des taux variables (voire des taux négatifs) selon l'empreinte carbone des différents produits : sous bénéfice d'une analyse plus approfondie, les activités de réparation, le marché de l'occasion (en prenant garde de ne pas prolonger des équipements énergivores) [Ceron et Baillon, 1979 ; Cooper, 2005], les travaux d'isolation, etc. pourraient en être exonérés.

- Elle est particulièrement adaptée aux « régimes fragmentés » de politiques climatiques qui ont de bonnes chances de durer, comme le suggèrent les négociations climatiques depuis Copenhague.

L'idée d'une «TVA carbone » comme forme plus politiquement acceptable du controversé «ajustement aux frontières » semble donc porteuse d'avenir. Olivier Godard pointe d'ailleurs le parallélisme des réflexions :

\footnotetext{
« Dans une perspective de long terme, impliquant une négociation nouvelle conjointement dans le cadre de l'OMC et celui de la convention-climat, il serait judicieux de voir dans les mécanismes d'ajustement aux frontières liés à l'émission de GES les bases d'un régime fiscal et commercial international analogue à celui qui a été adopté, non sans grincement, pour la TVA et les taxes sur la consommation [...] Ce serait la façon la plus économiquement rationnelle

2 «L'ajustement carbone sur les imports et les exports fonctionnerait comme la TVA pour laquelle les pays se sont accordés sur un régime d'ajustement aux frontières : les exportations sont détaxées puis retaxées dans le pays d'arrivée" (Olivier Godard). Voir http://www.journeeseconomie.org/ fichier/27_2.pdf.
}

d'intégrer les politiques climatiques dans les échanges commerciaux d'un monde dont les régions auraient à vivre durablement avec des prix du carbone sensiblement différents » (Godard et Ponssard, 2011, p. 85).

\section{Les limites}

Ces idées, pour intéressantes qu'elles soient, se heurtent à des limites théoriques et pratiques de taille :

- un problème d'adaptation des inventaires : s'il est relativement aisé de mesurer les ventes de carburant ou les émissions de procédés industriels, il est plus difficile de mesurer sur une base standardisée le carbone incorporé dans les différents produits échangés. Plus on s'éloigne de la source (principalement les ressources fossiles et leurs utilisations), plus les marges d'erreur et les interrogations sur le périmètre à prendre en compte augmentent. On imagine les années nécessaires pour redéfinir des standards d'inventaires ;

- la prise en compte des importations conduisant à alourdir la charge d'une grande partie des pays développés (Fig. 1), elle risque d'être peu acceptable politiquement. Ces pays cherchent déjà à se sortir du guêpier lié à la prise en compte de leur responsabilité historique (grandfathering) - en termes cumulatifs et depuis 1800, leur part dans les émissions est écrasante -, ils ne souhaitent évidemment pas se voir alourdir encore le fardeau. Ceci étant, dans une négociation, troquer une partie de l'effacement de la dette écologique contre la prise en compte du carbone importé pourrait contribuer à aborder plus raisonnablement l'avenir ;

- enfin et surtout, une approche de consommation ne règle pas, tant s'en faut, les problèmes d'équité. S'il peut sembler légitime de préconiser une certaine convergence des modes de vie, ou en tout cas de fixer des objectifs d'émissions par tête, d'autres enjeux d'équité se cachent derrière la consommation. Certains pays ou certains groupes sociaux (pays froids, populations éloignées) demanderaient des compensations pour leurs handicaps «naturels ", ou argueraient de la responsabilité historique des pays du Nord pour réclamer un «droit à consommer» au nom d'un rattrapage. Les débats et revendications ayant surgi en France au moment du projet de taxe carbone sont un exemple des situations inextricables dans lesquelles il est possible de se trouver.

\section{L'implication effective des ménages}

Au-delà de ces questions posées aux politiques climatiques nationales et internationales, d'autres outils concrets permettent une implication des ménages, que ces outils soient intégrés dans une vision plus large d'un régime climatique ou non. Ils paraissent en particulier adaptés à l'élaboration de politiques dans un cadre national ou régional que les développements des négociations internationales induisent. 


\section{La «version dure » : permis individuels négociables et cartes carbone}

Appréhender le niveau de la réduction des émissions de GES et la nécessité de responsabiliser les individus vis-à-vis de leurs émissions revient à la fois à les informer sur les conséquences de leurs choix et à leur demander de respecter une double contrainte budgétaire en termes monétaires et d'émissions.

Ce constat a donné lieu à un ensemble de travaux sur les budgets carbone individuels et les instruments de régulation associés (permis individuels négociables, cartes carbone, etc.).

L'acceptabilité sociale des politiques d'atténuation est un problème bien loin d'être résolu (Owen et al., 2008). L'objectif des évaluations axées sur la production était de fonder un marché du carbone où des droits d'émissions s'échangent entre les industries. Ceci se déroule à grande distance du citoyen et ne contribue nullement à le rendre conscient des enjeux et du fait que le carbone devra avoir un coût qui se répercutera sur lui dans un futur proche. Un résultat évident a été la levée de boucliers du public face à l'instauration de la taxe carbone en France, conduisant à un échec, même avec une assiette réduite et à un taux trop bas pour être efficace (Godard, 2010). Cet échec ne signifie pas que l'on serait arrivé à un résultat différent en proposant au consommateur de prendre directement ses responsabilités dans un contexte de rationnement des émissions. Il n'en reste pas moins que l'enjeu est bien là : rendre les ménages réellement responsables de leurs comportements et mettre dans leurs mains, de manière très explicite, les implications en matière d'émissions de leur choix de consommation et de comportements. D'où l'utilité d'une préfiguration des instruments possibles.

Le budget carbone individuel consiste en une attribution aux individus de droits d'émission, éventuellement négociables sur un marché. Le concept a fait l'objet de propositions et de recherches depuis maintenant une quinzaine d'années (Ayres, 1997; Fleming, 1998). L'essentiel de ces travaux provient du Royaume-Uni. Ils comportent des déclinaisons diverses :

- les Personal Carbon Allowances dont le champ d'application serait constitué des émissions directes des particuliers dues à leur consommation d'énergie pour le transport personnel et l'habitat (les émissions des personnes morales relevant d'autres instruments) [Fawcett, 2005];

- les Tradable Energy Quotas et Domestic Tradable Quotas (DTQ) qui embrasseraient les émissions des particuliers et des personnes morales. C'est de cette dernière catégorie que nous traitons ci-dessous (Starkey et Anderson, 2005).

Fondamentalement les DTQ sont un système de permis négociables destiné à réduire les émissions de GES provenant de l'usage de l'énergie. Les droits sont attribués et débités aux consommateurs finaux de produits pétroliers et d'électricité. Ils sont attribués gratuitement et de manière égalitaire, alors que les organisations les achètent sur un marché national du carbone. Les individus qui n'utilisent pas tout leur quota peuvent revendre le surplus sur le marché national et inversement d'autres peuvent acquérir des droits supplémentaires sur le même marché.

Il existe plusieurs variantes du concept, un fonctionnement possible a été décrit, notamment par le Tyndall Centre, au regard des dimensions suivantes (Starkley et Anderson, 2005) :

- l'allocation des droits. Elle se fait bien sur une base égalitaire (discussion toutefois concernant les ménages avec enfants), ce qui n'exclut pas des politiques complémentaires traitant des populations vulnérables (pauvres, ruraux, etc.). Il est considéré que l'attribution égalitaire favoriserait l'acceptation du système par les citoyens ;

- le réalisme. Il semble techniquement réaliste de s'appuyer sur l'infrastructure existante des cartes de crédit. Le travail propose toutefois des solutions adaptées aux individus réfractaires à ces moyens de paiement ;

- l'efficacité. Le coût de la mise en place (700 millions à 2 milliards de livres sterling) et du fonctionnement (1 à 2 milliards) d'un tel système est élevé (Lane et al., 2008), mais toutefois inférieur à celui d'autres projets gouvernementaux comme la mise en place d'une carte nationale d'identité ou d'un système de péage sur le transport routier (Starkey et Anderson, 2005, p. 4) ;

- l'organisation du marché (articulation entre particuliers, industriels, courtiers, etc.) ;

- le recyclage des fonds issus des mises aux enchères des quotas ;

- la protection de la vie privée ;

- la compatibilité avec le système européen de permis négociables, pour éviter les doubles comptes.

Des tests théoriques et en conditions réelles ont été menés sur des volontaires (Prescott, 2008), comme sur l'île de Norfolk en Australie, avec comme conclusion une bonne faisabilité du dispositif. Toutefois, après avoir fait effectuer un bilan des différents travaux, le gouvernement du Royaume-Uni a renoncé à mettre en œuvre un tel système (Lane et al., 2008).

D'autres débuts d'expérimentation, plus pragmatiques (Rousseaux, 2009) combinent application locale, objectifs moins contraignants, souvent sans marché de droits à polluer, attribution de récompenses et autres points verts, mutualisation possible des gains.

\section{La «version douce » : de la sensibilisation au passage à l'acte}

Des outils plus «doux » et sans doute plus adaptés à l'état actuel de la prise en charge de la question 
climatique dans nos sociétés existent. Ils regroupent tout un ensemble de démarches volontaires qui peuvent être mises en œuvre à l'échelle locale et individuelle.

Très visible, leur efficacité peut être surestimée par certains (les médias, les entreprises) ou au contraire négligée par d'autres (les macroéconomistes, les négociateurs climatiques).

Il faut sans doute restituer leur intérêt, pour plusieurs raisons.

- Travailler sur ces démarches volontaires permet de penser et de prendre en compte le levier comportemental (Bozonnet, 2007 ; Dobré et Juan, 2009 ; Shove, 2010), qui reste un grand mystère, et dont on sait pourtant qu'il constitue souvent la variable d'ajustement, permettant de «boucler» des scénarios de type facteur 4 . Par exemple, dans un travail sur la prospective de la mobilité de tourisme et de loisirs à l'horizon 2050 (Ceron et Dubois, 2006), nous avons montré qu'étant donné les perspectives de développement économique et la capacité réelle mais limitée de la technologie, le paramètre permettant d'atteindre de fortes réductions des émissions de GES du tourisme était un changement radical de la " culture du voyage », avec un renouveau du sens de la proximité, des modes de vie plus lents, un soin de l'environnement immédiat... sans qu'il soit possible d'identifier les leviers permettant effectivement de réduire la propension à voyager.

- Le franchissement de seuils comportementaux (tipping points) est une notion réelle, mais qui n'est observée qu'ex post (Vincent, 2010) et dont les facteurs explicatifs ne sont pas toujours compris. Les Français ont massivement accepté la limitation de vitesse sur la route et intégré ce comportement en routine même en l'absence de radars ; le taux de tri sélectif a bondi après des années de sensibilisation, en dépit des efforts que cela nécessite... En revanche, rien ne semble pouvoir décider les ménages à isoler leurs murs ou à réduire la température de leur logement en hiver.

Ces outils et démarches sont de plusieurs types. On note plusieurs tendances récentes :

- une sophistication et un développement rapide des outils Internet ;

- un passage de l'habitat au mode de vie dans son ensemble, mais aussi de l'énergie au carbone : la culture existante de maîtrise de la demande de l'énergie dans le bâtiment est en train de s'étendre à une problématique de changement climatique ;

- au-delà des essais de sensibilisation, un problème évident de passage à l'acte en l'absence d'autres leviers d'action. Les initiatives restent parfois confidentielles, ne prêchent que les convaincus, la communication n'est pas forcément adaptée au grand public (elle est culpabilisante, sentencieuse ou infantilisante...) [Centre d'analyse stratégique, 2011].

\section{Calculateurs et coaching carbone}

Les calculateurs individuels d'émissions de GES permettent une sensibilisation des ménages et pour certains un début de passage à l'acte quand le processus comprend un accompagnement de type coaching carbone ${ }^{3}$. La France dispose du bilan carbone personnel, le Coach Carbone $^{\circledR}$, développé en 2010 par l'Ademe. Des outils de même type ont été développés à l'étranger, ils sont nombreux et de caractéristiques très diverses. À l'origine de ces initiatives, on trouve des associations et des ONG, des entreprises commerciales (bureaux d'études, etc.), des universités et des organismes gouvernementaux.

Les associations et les ONG fournissent sauf exception des calculateurs grossiers, utilisables en un temps minimal dont le but paraît avant tout la sensibilisation. On entre déjà dans un niveau de précision supérieur avec les universités, mais ce sont sans doute les calculateurs « officiels » aux États-Unis et au Royaume-Uni qui fournissent les exemples les plus intéressants à comparer avec celui de l'Ademe. Après le diagnostic, ces trois outils débouchent sur une phase où ils proposent des solutions de réduction des émissions, chiffrées en termes physiques et monétaires.

Au-delà, la réflexion porte sur de nouveaux services, en ligne ou en face à face, d'accompagnement des ménages dans leurs efforts de réduction : c'est le «coaching carbone ».

\section{Écoguides et opérations pilotes}

On regroupe ici tout un ensemble d'opérations, menées à l'initiative de la société civile (ONG), des collectivités locales ou de l'État (Ademe, éducation nationale) et visant à développer l'engagement citoyen. On ne compte plus les « écogestes » et les « opérations écocitoyennes ». L'association Objectif climat a par exemple animé en Alsace des groupes d'actions locaux, avec 150 ménages volontaires pour réduire leur empreinte carbone. Là encore on note une montée en puissance du thème $\mathrm{du}$ changement climatique par rapport aux autres problématiques environnementales.

\section{La volonté d'impliquer les citoyens dans les politiques territoriales}

La loi Grenelle II a créé ou officialisé de nouveaux outils territoriaux de politiques climatiques : les schémas régionaux climat, air, énergie, qui ont été élaborés conjointement par l'État et les Régions, et les plans climat énergie territoriaux, sous la responsabilité des collectivités territoriales de plus de 50000 habitants.

3 www.air-climat.org, dossier sur le coaching carbone. 
L'implication des individus dans ces politiques peut se faire par deux moyens :

- au moment de leur élaboration par la mise en place d'une vraie démarche de participation. Cette concertation souvent mise en place par le maître d'ouvrage n'est cependant pas prévue par les textes. Au risque que ces politiques restent des démarches administratives, sans conviction et avec le simple objectif de remplir une obligation ;

- dans leur mise en œuvre, par le développement d'actions citoyennes, plus ou moins volontaires ou réglementaires, incitatives ou coercitives. Le "volet citoyen » des plans climat reste encore à inventer.

\section{Conclusion}

Ces dernières années ont montré un développement des travaux sur les liens entre consommation et carbone, à la fois avec l'amélioration des inventaires et avec le design d'outils de politique publique. Cet article a cherché à en faire la synthèse, tout en proposant un début d'analyse critique.

Sans forcément apporter toutes les réponses (et en posant d'autres questions), le fait d'introduire une approche de consommation dans les politiques climatiques a au moins deux intérêts : celui de réintroduire le ménage et le citoyen comme acteur (ou cible) des politiques climatiques ; et celui de pointer l'insuffisance des régimes climatiques actuels : problème des fuites de carbone, problèmes d'iniquité qui seront de plus en plus visibles au fur et à mesure que la contrainte se renforcera, manque d'attention au levier-clé des modes de vie, etc.

Dans les politiques climatiques actuelles, très influencées par la (macro) économie, les instruments de marché (taxes, permis négociables) sont fortement mis en avant, parfois au détriment des outils réglementaires classiques (normes, interdictions, etc.) ou des leviers comportementaux. Les approches de consommation permettent :

- sur un plan scientifique, de montrer que les individus ne sont pas que des homo economicus mais agissent aussi en fonction de ressorts psychoculturels parfois plus forts que leur rationalité économique ;

- sur le plan des politiques, de rappeler que tout ne se passe pas dans un monde idéal de marché dans lequel les ajustements seraient automatiques. Les marchés carbone pourraient avoir des effets pervers, à la fois en termes économiques (l'aviation pourrait racheter une part importante des permis et exclure de fait certaines industries de base de l'accès au mécanisme) et sociaux (inégalités et impact sur les populations les plus vulnérables par le biais des prix). Il est important de comprendre et d'anticiper ces effets, et de rappeler qu'il existe d'autres modes de régulation du changement climatique.
L'une des principales limites des approches de consommation, révélée par cet article, est qu'elles ne permettent pas de régler les enjeux d'équité qu'elles soulèvent. Réintroduire les importations dans les inventaires d'émissions, donner une vision plus large de l'empreinte carbone d'un mode de vie donné, irait sans doute dans le sens d'une plus grande transparence, mais ne fournit pas directement de clés pour l'action. Sur quels critères fixer les objectifs ? Comment attribuer les émissions à chaque pays ou à chaque groupe social? Ces questions continuent d'alimenter des débats qui font appel à des valeurs nécessairement subjectives et à des jeux d'intérêts qu'il n'appartient pas aux scientifiques de trancher. Ces derniers peuvent cependant fournir les bases de débats plus rigoureux. Donner un éclairage de consommation sur la problématique des émissions de gaz à effet de serre y contribue.

\section{Références}

Aall, C., Hille, J., 2009. Consumption: A missing dimension in climate policy, in Bhaskar, R., Frank, C., Høyer, K.G., Naess, P., Parker, J., Interdisciplinarity and Climate Change, London, Routledge.

Agence fédérale de l'environnement allemande (Umweltbundesamt), 2009. A climate-neutral lifestyle: Consumers lead the way in climate protection (online: http:/ / www.umweltbundesamt).

Aichele, R., Felbermayr, G., 2012. Kyoto and the carbon footprint of nations, Journal of Environmental Economics and Management, 63, 3, 336-354.

Anderson, K., Bows, A., Mander, S., Shackley, S., Agnolucci, P., Ekins, P., 2006. Decarbonising Modern Societies: Integrated Scenarios Process and Workshops, Norwich (UK), Tyndall Centre.

Assemblée nationale, 2014. Projet de loi relatif à la transition énergétique pour une croissance verte, Paris.

Ayres, R., 1997. Environment market failures: Are there any local market-based corrective mechanisms for global problems?, Mitigation and Adaptation Strategies for Global Change, 1, 3, 289-309.

Babiker, M.H., 2005. Climate change policy, market structure, and carbon leakage, Journal of International Economics, 65, 2, 421-445.

Bonduelle, A., Szuba, M., Zuindeau, B., 2011. Facteur 4: le chantier social et politique, Revue Développement Durable et Territoires, 2, 1.

Bows, A., Mander, S., Bows, A., Mander, S., Starkey, R., Bleda, M., Anderson, K., 2006. Living with a Carbon Budget, Manchester, Tyndall Centre.

Bows, A., Barrett, J., 2010. Cumulative emission scenarios using a consumption-based approach: A glimmer of hope?, Carbon Management, 1, 161-175.

Bozonnet, J.-P., 2007. De la conscience écologique aux pratiques : comment expliquer le hiatus entre attitudes environnementalistes et comportements, Grenoble-Toulouse, Pacte IEP Grenoble-Toulouse. 
Centre d'analyse stratégique, 2011. Nudges verts: de nouvelles incitations pour des comportements écologiques. Note d'analyse 216.

Ceron, J., Baillon, J., 1979. La Société de l'éphémère, Grenoble, Paris, Presses universitaires de Grenoble, Éditions de la Maison des sciences de l'Homme.

Ceron, J.-P., Dubois, G., 2006. Demain le voyage : La mobilité de tourisme et de loisirs des français face au développement durable. Scénarios à 2050. Ministère des Transports, de l'Équipement, du Tourisme et de la Mer, Paris.

Ceron, J.-P., Dubois, G., de Torcy, L., 2010. Développement touristique de l'outre-mer et dépendance au carbone, Paris, Marseille, TEC, AFD.

CGDD, 2010. $\mathrm{CO}_{2}$ et activités économiques de la France : tendances 1990-2007 et facteurs d'évolution. Commissariat général au développement durable, Paris.

Chakravartya, S., Chikkaturb, A., de Coninckc, H., Pacalaa, S., Socolowa, R., Tavoni, M., 2009. Sharing global $\mathrm{CO}_{2}$ emission reductions among one billion high emitters, PNAS, 106, 11884-11888.

Cooper, T., 2005. Slower consumption reflections on product life spans and the "throwaway society", Journal of Industrial Ecology, 9, 1-2, 51-67.

Davis, S.J., Caldeira, K., 2010. Consumption-based accounting of $\mathrm{CO}_{2}$ emissions, Proceedings of the National Academy of Sciences, 107, 12, 5687-5692.

Dobré, M., 2012. Mettre en scénario les modes de vie en 2050. Communication au colloque Usages de l'énergie dans les bâtiments : penser la transition, Paris, ESIEE, 19-20 janvier.

Dobré, M., Juan, S. (Eds), 2009. Consommer autrement : la réforme écologique des modes de vie, Coll. Sociologies et environnement, Paris, L'Harmattan.

Edgar, G.H., Glen, P.P., 2009. Carbon footprint of nations: A global, trade-linked analysis, Environmental Science and Technology, 43, 16, 6414-6420.

EIFER/Sociovision, 2011. Milieux urbains durables : prospective des modes de vie dans des villes post-carbone. Ademe, Paris.

Eriksson, M., 2008. Konsumtionens klimatpåverkan, Stockholm, Naturvårdsverket.

Fawcett, T., 2005. Personal carbon allowances: Background document $L$ for the $40 \%$ house report. Environmental Change Institute, University of Oxford, Oxford.

Finke, M., Gautier, C., 2013. Les émissions importées : le passager clandestin $d u$ commerce mondial, Réseau Action Climat France (http://www.rac-f.org/IMG/pdf/EMISSIONSIMPORTEES_RAC-Ademe-Citepa.pdf).

Fleming, D., 1998. Domestic tradable quotas as an instrument to reduce carbon dioxyde emissions. Proceedings of workshop, EUR18541, Bruxelles, European Commission, 1-2 july.

Frese, S.D., Bang, J.K., Nordbo, J., 2008. Dansk forbrug, global forurening En analyse af Danmarks CO2-fodaftryk med særligt fokus på Kina, Copenhagen, WWF.

Gallopin, G., Hammond, A., Raskin, P., Swart, R., 1997. Branch points: Global scenarios and human choice. Polestar series report. Stockholm Environment Institute, Stockholm.

Godard, O., 2010. Genèse et avortement de la contribution carbone en France (2009-2010), Cahier de la Chaire Business Economics de l'École Polytechnique, Palaiseau.
Godard, O., Ponssard, O. (Eds), 2011. Économie du climat : pistes pour l'après-Kyoto, Palaiseau, Éditions de l'Ecole Polytechnique.

Hansen, J., Sato, M., Kharecha, P., Beerling, D., Berner, R., Masson-Delmotte, V., Pagani, M., Raymo, M., Royer, D.L., Zachos, J.C., 2008. Target atmospheric $\mathrm{CO}_{2}$ : Where should humanity aim?, The Open Atmospheric Science Journal, 2, 217-231.

Helm, D., Smale, R., Phillips, J., 2007. Too Good To Be True? The UK's Climate Change Record, Oxford, New College, University of Oxford.

Hong, L., Pei Dong, Z., Chunyu, H., Gang, W., 2007. Evaluating the effects of embodied energy in international trade on ecological footprint in China, Ecological Economics, 62, 136-148.

IPCC, 1996. Revised 1996 IPCC: Guidelines for national greenhouse gas inventories. Intergovernmental Panel on Climate Change, Geneva.

IPCC, 2001. Climate change 2001: Synthesis report. Cambridge University Press, Cambridge (UK).

IPCC, 2014. Climate change 2014: Mitigation of Climate Change. Cambridge University Press, Cambridge (UK).

Kanemoto, K., Lenzen, M., Peters, G.P., Moran, D.D., Geschke, A., 2012. Framework for comparing emissions associated with production, consumption and international trade, Environmental science and technology, 46.

Kates, R., Leiserowitz, A., Parris, T., 2006. Great transition values: Present attitudes, future changes. GTI paper series, Tellus Institute, Boston.

Lane, C., Harris, B., Roberts, S., 2008. An analysis of the techniqcal feasability and potential cost of a personal carbon trading scheme. Accenture and Centre for sustainable Energy/DEFRA, London.

Lenglart, F., Lesieur, C., Pasquier, J.-L., 2010. Les émissions de $\mathrm{CO}_{2}$ du circuit économique en France. Service de l'observation et des statistiques (SOeS), Commissariat général au développement durable, Paris.

Mäenpää, I., Siikavirta, H., 2007. Greenhouse gases embodied in the international trade and final consumption of Finland: An input-output analysis, Energy Policy, 35, 128-143.

Meinhausen, M., 2006. What does a $2{ }^{\circ} \mathrm{C}$ targetmean for greenhouse gas concentrations: A brief analysis based on multi gas emission pathways and several climate sensitivity uncertainty estimates, in Schellnhuber, H.J., Cramer, W., Nakicenovic, N. Wigley, T., Yohe, G. (Eds), Avoiding dangerous climate change, Cambridge (USA), Cambridge University Press.

Munasinghe, M., Dasgupta, P., Southerton, D., Bows, A., McMeekin, A., 2009. Consumers, Business and Climate Change, Manchester, The University of Manchester Sustainable Consumption Institute.

Muñoz, P., Steininger, K.W., 2010. Austria's $\mathrm{CO}_{2}$ responsibility and the carbon content of its international trade, Ecological Economics, 69, 10, 2003-2019.

Owen, L., Edgar, L., Prince, S., Doble, C., 2008. Personal carbon trading: Public acceptability. A research report completed to the Department for environment, food and rural affairs. DEFRA, London. 
Parry, M., Palutikof, J., Hanson, C. Lowe, J., 2008. Climate policy: Squaring up to reality, Nature Reviews: Climate Change, 2, 68-70.

Pasquier, J.-L., 2010. Les comptes physiques de l'environnement, une base pour de nouveaux indicateurs sur l'interface économie environnement : le cas des émissions de $\mathrm{CO}_{2}$ de la France, $L a$ Revue du CGDD, Service de l'observation et des statistiques (SOeS) du Commissariat général au développement durable.

Peters, G.P., Hertwich, E.G., 2006. Pollution embodied in trade: The Norwegian case, Global Environmental Change, 16, 4, 379-387.

Peters, G.P., Hertwich, E.G., 2008. Post-Kyoto greenhouse gas inventories: Production versus consumption, Climatic Change, 86, 1-2, 51-66.

Peters, G.P., Minx, J.C., Weber, C.L., Edenhofer, O., 2011. Growth in emission transfers via international trade from 1990 to 2008, Proceedings of the National Academy of Sciences, $108,8903-8908$.

Prescott, M.A., 2008. A persuasive climate: Personal trading and changing lifestyles, London, The Royal Society for the Encouragement of Arts, Manufactures and Commerce.

Radanne, P., 2004. La division par 4 des émissions de carbone en France d'ici 2050. Mission interministérielle de l'effet de serre, Paris.

Raskin,P.,Monks, F., Ribeiro, T., van Vuuren, D.,Zurek,M., 2005. Global scenarios in an historical perspective, in Carpenter, S.R., Pingali, P.L., Bennett, E.M., Zurek, M.B., Ecosystems and Human Well-being. Scenarios: Findings of the Scenarios working group of the Millenium Ecosystem Assessment, Washington D.C., Island Press, 35-44.

Rosen, R., Electris, C., Raskin, P., 2010. Global scenarios for the century ahead, Boston, Tellus Institute.

Rousseaux, S., 2009. État des lieux international des programmes de " carte carbone » pour les particuliers. Ademe, Paris.
Shove, E., 2010. Beyond the ABC: Climate change policy and theories of social change, Environment and Planning A, 42, 6, 1273-1285.

SPREAD, 2012. Future Scenarios for New European Social Models with Visualisations. Wuppertal (Germany), UNEP/Wuppertal Institute Collaborating Centre on Sustainable Consumption and Production (online: http://www.sustainablelifestyles.eu/fileadmin/images/content/ D4.1_FourFutureScenarios.pdf).

Starkey, R., Anderson, K., 2005. Domestic Tradable Quotas: A Policy for Reducing Greenhouse Gas Emissions from Energy Use, Norwich (UK), Tyndall Centre.

Steckel, J.C., Kalkuhl, M., Marschinski, R., 2010. Should carbon-exporting countries strive for consumption-based accounting in a global cap-and-trade regime?, Climatic Change, 100, 3, 779-786.

Subak, S., 1995. Methane embodied in the international trade of commodities, implications for global emissions, Global Environmental Change, 5, 5, 433-446.

Vincent, S., 2010. De l'autostop au covoiturage : institutionnalisation d'une pratique, Revue Urbanisme, 370.

Weber, C.L., Matthews, H.S., 2008. Quantifying the global and distributional aspects of American household carbon footprint, Ecological Economics, 66, 2-3, 379-391.

Wiedmann, T., Wood, R., Lenzen, M., Minx, J., Guan, D., Barrett, J., 2008. Development of an embedded carbon emissions indicator - Producing a time series of input-output tables and embedded carbon dioxide emissions for the UK by using a MRIO Data Optimisation System. Report to the UK Department for Environment, Food and Rural Affairs by Stockholm Environment Institute at the University of York and Centre for Integrated Sustainability Analysis at the University of Sydney, DEFRA, London.

Wiedmann, T., Wood, R., Minx, J., Lenzen, M., Guan, D., Harris, R., 2010. A carbon footprint time series of the UK: Results from a multi-region input output model, Economic Systems Research, 22, 1, 19-42. 